\title{
New Concept of Small Molecules Interaction with Proteins - An Application to Potential COVID-19 Drugs
}

\author{
Irena Cosic ${ }^{1}$, Drasko Cosic ${ }^{1}$, Ivan Loncarevic ${ }^{2}$
}

\author{
${ }^{1}$ AMALNA Consulting, Black Rock, 3193, Australia \\ ${ }^{2}$ QuantBioRes - QBR A/S, Copenhagen, 2860, Denmark
}

Running Title: New Concept for COVID-19 Drugs

\begin{abstract}
With the huge demand for new effective drugs there is large need for computational methods capable of quick preselection of potential drugs before they are chemically and biologically tested. Particularly, with the latest outbreak of coronavirus SARS-CoV-2 causing COVID-19 pandemic, there is urgent need to preselect number of potential small molecule drugs that are already approved for other purposes and are clinically tested on infected people with variety of success. The already established Resonant Recognition Model (RRM) proposes that selectivity of biological interactions and functions between proteins, DNA/RNA, is based on resonant electromagnetic energy between interacting macromolecules at the specific frequency for the specific interaction/function. However, this approach cannot be applied for selective specific interactions between proteins and small molecules (potential drugs), as small molecules are not linear sequential molecules. Here, we extended the RRM model to small molecules interaction with proteins proposing that energy frequencies of free electrons in small molecules are the most relevant for their resonant recognition and interaction with proteins. This extended RRM model is firstly tested here with couple of natural examples to explain and support the model, following with application to already approved drugs, which are also potential COVID-19 drugs including remdesivir, chloroquine and hydroxychloroquine. This newly extended RRM model opens new avenues for biochemistry and pharmaceutical industry in analyzes of small molecule - protein interaction, and consequently in preselection of new drugs and drug design in general.
\end{abstract}

Keywords: Drug Design, Small Molecules, Resonant Energy, Bioelectromagnetism, Resonant Recognition Model, SARS-Cov-2, COVID-19, Hydroxychloroquine, Chloroquine, Remdesivir

\section{Introduction}

With the huge developments within pharmaceutical industry towards finding the whole variety of effective drugs (small molecules), there is large demand for pre-selective computational methods capable of quick preselection of small molecules before they are chemically and biologically tested. Such quick preselection method would significantly narrow down number of potential molecular candidates that would need to be experimentally tested and thus would enormously save resources, time and costs involved in discovery of new drugs, and consequently would provide quicker and better health outcomes. One of examples could be preselection of possible drugs for COVID-19.

SARS-CoV-2 virus is rapidly growing viral infection causing coronavirus disease COVID-19, which has spread all around the world as pandemic with enormous number of people infected and large number of fatalities. With such pandemic there is major need and rush to find either cure or vaccine. One of the quickest approaches is to test existing already approved and safe drugs for possibility of their activity against SARS-CoV-2 virus. Along those lines, it has been quoted in reference [1]: "Hydroxychloroquine and chloroquine have garnered unprecedented attention as potential therapeutic agents against COVID-19 following several small clinical trials, uncontrolled case series and public figure endorsement". Those two drugs have been labeled as potential game changers, although the mechanisms of their activity are not yet known [2]. Chloroquine has been extensively used for prevention and treatment of malaria, as well as the treatment of autoimmune conditions such as rheumatoid arthritis and systemic lupus [3-4], while hydroxychloroquine was later introduced due to its superior safety profile [4]. In vitro and in vivo experimental data for the use of hydroxychloroquine and chloroquine against

This article is published under the terms of the Creative Commons Attribution License 4.0 Author(s) retain the copyright of this article. Publication rights with Alkhaer Publications. Published at: http://www.ijsciences.com/pub/issue/2020-09/

DOI: 10.18483/ijSci.2390; Online ISSN: 2305-3925; Print ISSN: 2410-4477 
SARS-CoV-2 so far have been summarized in review article [1]. Although hydroxychloroquine and chloroquine have been already successfully tested in number of clinical trials with COVID-19 patients [2], the mechanism of their actions against SARS-CoV-2 virus are not yet known. In addition, remdesivir drug as small molecule, which has been previously approved for treatment for number of retroviruses, has been recently also approved for treatment of COVID-19 in some countries. Remdesivir inhibits production of viral RNA, but the mechanism of how it is happening is not yet well explained. To analyze interaction between small molecules as potential drugs and related proteins, we are introducing here the extended version of the Resonant Recognition Model (RRM), which is based on resonant electromagnetic energy transfer between interacting biomolecules.

We have previously established Resonant Recognition Model (RRM) for analyzes of protein interactions with other proteins, DNA/RNA, which takes into account functionality of those macromolecules and is based on periodicities (frequencies) within distribution of free electron energies along these macromolecules [5-17]. With electron charge moving through protein, DNA/RNA backbone passing different free electron energies within different side groups (amino acids or nucleotides) electromagnetic radiation or absorption of frequency related to distribution of energies of free electrons along macromolecular backbone will be produced. The RRM model considers long distance recognition, as it proposes that these electromagnetic frequencies characterize specificity of recognition and interaction between protein, DNA/RNA and their targets through resonant energy transfer on distance between interacting macromolecules.

However, this already established RRM approach cannot be applied for interactions between proteins and small molecules, as small molecules are not linear sequential molecules. Still, we propose that small molecules also recognize on the distance and interact with proteins through electromagnetic resonant energy transfer enabling specific biological activity. To expand the idea of electromagnetic resonant recognition to small molecules and their interaction with proteins, we have here extended RRM model by calculating electromagnetic frequencies of free electron energies within the small molecule and comparing these frequencies with RRM characteristic frequencies for relevant interacting proteins.

This manuscript is aimed to present for the first time new biophysical model and calculations for interaction between small molecules and proteins.
We firstly test the extended RRM model with couple of natural examples to explain and support these calculations, following with application to already approved drugs, which are also potential COVID-19 drugs including remdesivir, chloroquine and hydroxychloroquine for their ability to interact with viral proteins and thus interfere with viral infection. The extended RRM model, as new concept, presented within this manuscript can be used in large number of examples in pharmacology and biotechnology.

\section{Methods}

\section{Resonant Recognition Model (RRM)}

The Resonant Recognition Model (RRM) is biophysical model that can analyze interaction and recognition between proteins and their protein, DNA/RNA targets [5-12]. The RRM model is based on the findings that certain periodicities (frequencies) within the distribution of energy of delocalized electrons along protein, DNA/RNA molecule are critical for protein, DNA/RNA biological function and/or interaction with their targets. If charge transfer through these macromolecules is introduced, then charge moving through macromolecular backbone can produce electromagnetic radiation, absorption and resonance with spectral characteristics corresponding to energy distribution.

The RRM model enables for these spectral characteristics to be calculated by assigning each amino acid physical parameter representing the energy of delocalized electrons of each amino acid. Comparing Fourier spectra of this energy distributions by using cross-spectral function, it has been found that proteins sharing the same biological function/interaction share the same periodicity (frequency) within energy distribution along the macromolecule. Furthermore, it was shown that interacting proteins and their targets share the same characteristic frequency, but opposite phase [5-12]. Thus, it has been proposed that the RRM frequencies characterize, not only general function, but also recognition and interaction between the specific macromolecule and its target, which then can be considered as resonant recognition [5-12]. This could be achieved through resonant energy transfer on distance between the interacting macromolecules through oscillations of electromagnetic field. Since there is evidence that proteins, DNA/RNA have certain conducting or semi-conducting properties, electrical charge moving through the macromolecular backbone and passing different energy stages caused by different amino acid or nucleotide side groups, can produce sufficient conditions for specific electromagnetic radiation or absorption. The frequency range of this field depends on charge velocity and distance between side groups. The RRM proposes that charge is travelling through 
macromolecular backbone at the velocity estimated at $7.87 \times 10^{5} \mathrm{~m} / \mathrm{s}$ [5-12]. For this velocity and the distance between amino acids in protein backbone, which is $3.8 \AA$, the frequency range obtained for protein interactions was estimated to be in the range of $10^{13} \mathrm{~Hz}$ up to $10^{15} \mathrm{~Hz}$. Therefore, the estimated range for both amino acid and nucleotide macromolecules includes far infra-red, infra-red and visible up to ultra-violet light spectrum.

To support this idea, we have compared our computational predictions with number of published experimental results [5-9,13-17]. These comparisons have shown strong linear correlation between frequencies, as calculated using the RRM model and experimentally measured characteristic frequencies, with the slope factor of $K=201$ [5-9,13-17]. This correlation can be represented as following:

$\lambda=\mathrm{K} / \mathrm{f}_{\mathrm{rrm}}$

where $\lambda$ is the wavelength of light radiation in nanometres $(\mathrm{nm})$, which can influence particular biological process, $\mathrm{f}_{\text {rrm }}$ is RRM numerical frequency and $\mathrm{K}$ is coefficient of this linear correlation.

The RRM concept, proposing that specific frequencies of electromagnetic radiation are critical for macromolecular activities and interactions, has been experimentally tested on electromagnetic frequencies activating l-lactate dehydrogenase [18], photon emission from dying melanoma cells [19], photon emission from lethal and non-lethal Ebola strains [20], JAK-STAT signalling pathway [21], as well as more recently on osteoblastic differentiation of stem cells by photo bio-modulation [17]. Even more, the RRM model, for the first time, explains how and why external blue light can be used in treatment of Crigler-Najjar syndrome [15]. This means that, by radiating the whole body with specific RRM frequency, the desired health and medical effects can be achieved.

Bearing all this in mind, we propose that the RRM concept is an excellent predictor for proteins and DNA/RNA selective interactions, biological processes, and pathways in living cells. In our previous work, we have calculated large number of specific frequencies for different protein and DNA/RNA biological functions and interactions [517].

\section{Extended Resonant Recognition Model for Small Molecules}

While our previously established Resonant Recognition Model (RRM), is able to calculate resonant recognition and interaction frequencies for linear macromolecules like proteins, DNA/RNA, the biggest challenge is how to calculate resonant recognition and interaction frequencies between small molecules and linear macromolecules like proteins. It is well known that small molecules also selectively interact with proteins and thus, we have extended the idea of Resonant Recognition Model (RRM) to small molecules proposing that such selective interactions between small molecules and proteins are also based on resonant energy transfer. For that purpose, we propose that energies of free electrons in small molecules are the most relevant for such resonant energy transfer. The energies of free electrons within small molecules can be calculated as the electron-ion interaction pseudo-potential (EIIP) of small molecule using the following semi-empirical formula as developed by Veljkovic [22-24]:

$<\mathrm{k}+\mathrm{q}|\mathrm{w}| \mathrm{k}>=0.25 \times \mathrm{Z} \times \sin (\pi \times 1.04 \times \mathrm{Z}) /(2 \times \pi)$

where $\mathrm{q}$ is change of momentum of delocalized electron in the interaction with potential $w$ (EIIP) in Rydberg $\left(\mathrm{Ry}=2.18 \times 10^{-18}[\mathrm{~J}]\right)$, while $\mathrm{Z}$ is average valence number over the whole small molecule as:

$\mathrm{Z}=(\Sigma \mathrm{Zi}) / \mathrm{N}$

where $\mathrm{Zi}$ is the number of valence electrons of the $\mathrm{i}$ th component of small molecule and $\mathrm{N}$ is the total number of atoms in the amino acid.

Once when energy of free electrons within small molecules have been calculated, we have used Einstein's equation to calculate frequencies related to these energies:

$E=h \times f$

where $\mathrm{E}$ is energy $[\mathrm{J}]$ of free electrons within small molecule, $\mathrm{h}\left(\mathrm{h}=6.626 \times 10^{-34}[\mathrm{Js}]\right)$ is Planck constant and $\mathrm{f}$ is corresponding frequency $[1 / \mathrm{s}]$.

As biological processes and related molecular interactions are not occurring in vacuum, but within biological tissue with different refractive indexes, it is more appropriate to use de Broglie formula as follows:

$\lambda=(\mathrm{h} \times \mathrm{c}) / \mathrm{E}$ in vacuum, where $\mathrm{c}$ is speed of light (c $\left.=2.998 \times 10^{8}[\mathrm{~m} / \mathrm{s}]\right)$

$\lambda=(\mathrm{h} \times \mathrm{v}) / \mathrm{E}$ in other materials, where $\mathrm{v}$ is speed of light in other materials

where $\lambda$ is wavelength of light, $\mathrm{h}$ is Planck constant, $\mathrm{E}$ is energy of free electrons within small molecule.

To calculate $\lambda$ in $[\mathrm{nm}]$ the speed of light in $[\mathrm{nm}]$ is c $=2.998 \times 10^{17}[\mathrm{~nm} / \mathrm{s}]$. To take into account that 
energy of free electrons within small molecules is calculated in $[\mathrm{Ry}]$ this energy must be presented into $[\mathrm{J}]$ as follows:

$\mathrm{E}[\mathrm{J}]=\mathrm{E}[\mathrm{Ry}] \times 2.18 \times 10^{-18}[\mathrm{~J} / \mathrm{Ry}]$

Thus, for vacuum:

$\lambda[\mathrm{nm}]=\left(6.626 \times 10^{-34}[\mathrm{Js}] \times 2.998 \times 10^{17}[\mathrm{~nm} / \mathrm{s}]\right) /$ $\left(\mathrm{E}[\mathrm{Ry}] \times 2.18 \times 10^{-18}[\mathrm{~J} / \mathrm{Ry}]\right)$

All biological processes and interactions are taking place within biological materials and thus speed of light will depend on refraction index within biological materials:

$\mathrm{v}=\mathrm{c} / \mathrm{n}$

where $\mathrm{n}$ is refraction index of biological materials.

For water refraction index is 1.33 , while for biological materials refraction index is: for cell membranes 1.46-1.60, for cytoplasm 1.35-1.39 and for proteins 1.36-1.55 [25]. Thus, for biological materials wavelength of light $\lambda_{b}$ is:

$\lambda_{\mathrm{b}}[\mathrm{nm}]=\left(6.626 \times 10^{-34}[\mathrm{Js}] \times 2.998 \times 10^{17}[\mathrm{~nm} / \mathrm{s}]\right) /$ (n x E[Ry] x $\left.2.18 \times 10^{-18}[\mathrm{~J} / \mathrm{Ry}]\right)$

where $\mathrm{n}$ is refraction index of biological materials. As stated above the refraction index of biological materials is within the range of 1.35-1.60 [25].

Bearing in mind all the above, we hypothesize here that wavelengths (frequencies) produced by energies of free electrons within small molecules are critical for small molecule biological functions and their recognition and interaction with proteins. We propose here that small molecules are recognizing and interacting with proteins through resonance at the same characteristic frequency (wavelength). To be able to compare resonant frequencies of small molecules, with resonant RRM frequencies, we can convert $\lambda_{b}[\mathrm{~nm}]$ into the RRM frequency, as related $\mathrm{RRM}$ frequency for small molecules can be presented using the formula below:

$\mathrm{f}_{\mathrm{sm}}=\mathrm{K}[\mathrm{nm}] / \lambda_{\mathrm{b}}[\mathrm{nm}]$

where $\mathrm{K}=201$ is coefficient in [nm] previously semiempirically identified to characterize the relationship between RRM frequencies and related electromagnetic radiation wavelengths, as explained above [5-8].

Thus, the RRM resonant frequency of small molecules can be calculated as follows:

$\mathrm{f}_{\mathrm{sm}}=(\mathrm{K} \times \mathrm{E}) /(\mathrm{h} \times(\mathrm{c} / \mathrm{n}))$ where: $f_{s m}$ is numerical RRM frequency corresponding to electromagnetic radiation resonances due to energies of free electrons within small molecules, $\mathrm{K}=201$ is coefficient in $\mathrm{nm}$ previously semi empirically identified to characterize the relationship between RRM frequencies and related electromagnetic radiation wavelengths, $\mathrm{E}$ is energy of free electrons within small molecules, $\mathrm{h}$ is Planck constant, $\mathrm{c}$ is speed of light, $\mathrm{n}$ is refraction index in biological materials.

The hypothesis, that wavelengths (frequencies) produced by energies of free electrons within small molecules are critical for small molecules biological functions and their recognition and interaction with proteins, has been tested here in couple of natural examples and applied to potential COVID-19 drugs.

\section{Results}

\section{Interaction between Cannabinoids and} Cannabinoid Receptors

Cannabinoid receptors as part of the endocannabinoid system are involved in control of number of physiological processes including appetite, pain and pleasure sensation, immune system, mood, and memory [26]. Cannabinoid receptors are activated by interaction with small molecules from three major groups of ligands: endocannabinoids (produced by the mammalian organisms), plant cannabinoids, and synthetic cannabinoids [27]. There are two main subtypes of cannabinoid receptors: $\mathrm{CB} 1$ and $\mathrm{CB} 2$, which are expressed in different tissue types [27]. However, there are additional receptors that can selectively bind to cannabinoids, for example: GPR55, which is produced within brain and TRPV1, which acts as ionotropic endocannabinoid receptor with central neuro modulatory effects. We have analyzed all cannabinoid receptors, as well as cannabinoid receptor suppressor CNRIP1, with the aim to find out the RRM characteristics related to cannabinoid biological function.

To achieve above, we have used the RRM model to analyze cannabinoid receptors and related suppressor, with the aim of finding out which electromagnetic resonant frequencies are characterizing interaction between cannabinoids as small molecules and related cannabinoid receptors as proteins. To find out the RRM characteristic frequency characterizing the biological function of cannabinoid receptors, we have analyzed, using RRM cross-spectral function, cannabinoid receptors: CB1, CB2, GPR55, TRPV1 and CNRIP1 suppressor proteins, from UniProt database, as listed in Appendix. The most prominent common frequency has been found at $\mathrm{fn}=0.3174 \pm 0.0024$, as presented in Figure 1, 
characterizing cannabinoid receptors biological function, which is activated by interaction with cannabinoid small molecules.

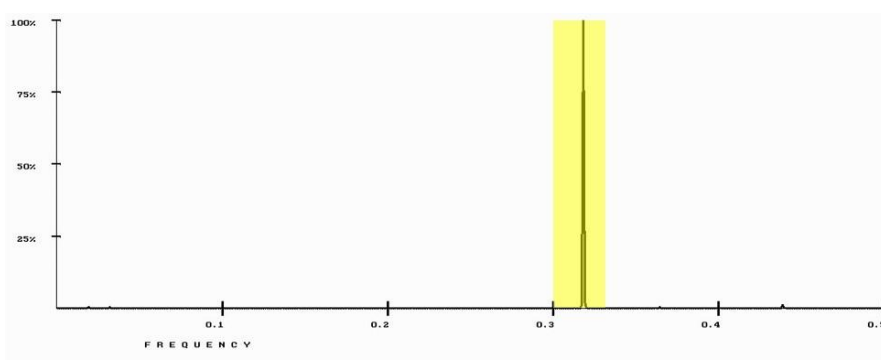

Figure 1. RRM cross-spectrum of cannabinoid receptors and suppressor proteins. The common RRM characteristic frequency is at $\mathrm{fn}=0.3174 \pm 0.0024$. RRM frequency range for cannabinoid small molecule between 0.30-0.33 highlighted in yellow.

On the other hand, using the chemical formula for cannabinoid small molecule $\left(\mathrm{C}_{21} \mathrm{H}_{30} \mathrm{O}_{2}\right)$, we have calculated energy of free electrons within cannabinoid small molecule to be at En=0.0942Ry. For this energy and refraction index for cell membranes (receptors are within cell membrane), between 1.46-1.60 [25], we have calculated the corresponding RRM frequency for cannabinoid small molecule to be between 0.30-0.33, as highlighted in yellow in Figure 1. All intermediate calculations for cannabinoid small molecule using formulas presented within Methods section have been presented within Table 1. Strikingly, this RRM frequency range for small molecule (cannabinoid) overlaps characteristic RRM frequency for related receptors, which is at $\mathrm{fn}=0.3174 \pm 0.0024$. As there is common frequency between characteristic RRM frequency of cannabinoid receptors and RRM frequency for cannabinoid small molecules, we propose that frequency $\mathrm{fn}=0.3174 \pm 0.0024$ is characterizing interaction between cannabinoids as small molecules and related cannabinoid receptors as proteins. Such result confirms our hypothesis that interaction between small molecules and related receptors/proteins is based on resonant electromagnetic recognition at specific frequency.

\section{Interaction between Kainic Acid and Glutamate Kainic Receptors}

Glutamate receptors exist mostly in the central nervous system on the membranes of neuronal and glial cells, as synaptic and non-synaptic receptors [28]. They are activated by binding to glutamate released into the synaptic cleft by presynaptic cells. There are number of experiments showing variety of nerve cells that express glutamate receptors and their interaction with glutamate $[29,30]$. Kainate receptors, as subgroup of glutamate receptors are classified as non-NMDA-type receptors and they are selectively activated by kainic acid (kainate). Kainate is potent neuroexcitatory small molecule that acts by activating glutamate receptors [31].

We have used the RRM model to analyze glutamate kainic receptors, with the aim of finding out which electromagnetic resonant frequencies are characterizing interaction between kainic acid (kainate) as small molecules and related glutamate kainic receptors as proteins. To find out the RRM characteristic frequency characterizing the biological function of glutamate kainic receptors, we have analyzed, using RRM cross-spectral function, glutamate kainic receptors, from UniProt database, as listed in Appendix. The most prominent common frequency has been found at $\mathrm{fk}=0.1143 \pm 0.0008$, as presented in Figure 2, characterizing glutamate kainic receptors biological function, which is activated by interaction with kainic acid (kainate) small molecules.

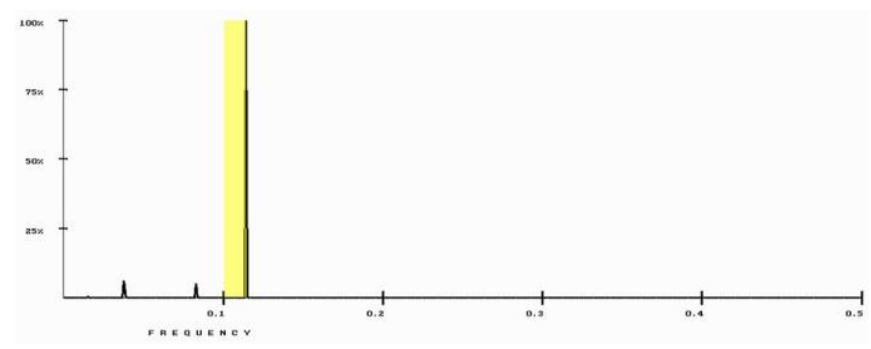

Figure 2. RRM cross-spectrum of glutamate kainic receptors. The common RRM characteristic frequency is at $\mathrm{fk}=0.1143 \pm 0.0008$. RRM frequency range for kainic small molecule between $0.10-0.11$ highlighted in yellow.

On the other hand, using the chemical formula for kainic acid $\left(\mathrm{C}_{10} \mathrm{H}_{15} \mathrm{NO}_{4}\right)$ as small molecules, we have calculated energy of free electrons within these small molecules to be at Ek=0.0304Ry. For this energy and refraction index for cell membranes (receptors are within cell membrane), between 1.46-1.60 [25], we have calculated the corresponding RRM frequencies to be for kainic small molecule between $0.10-0.11$, as highlighted in yellow in Figure 2. All intermediate calculations for kainate small molecule using formulas presented within Methods section have been presented within Table 1. This RRM frequency range for small molecules (kainic) overlaps characteristic RRM frequency, with marginal error of calculation, for related receptors at $\mathrm{fk}=0.1143 \pm 0.0008$. As there is common frequency between characteristic RRM frequency of glutamate kainic receptors and RRM frequency for kainic small molecules, we propose that frequency 
$\mathrm{fk}=0.1143 \pm 0.0008$ is characterizing interaction between kainic acid (kainate) as small molecules and related glutamate kainic receptors as proteins. Such result confirms again our hypothesis that interaction between small molecules and related receptors/proteins is based on resonant electromagnetic recognition at specific frequency.

\section{Interaction between Remdesivir and Retroviral Protease Proteins}

So far, we have shown, in examples above, the possibility that interaction between small molecules and related receptors/proteins is based on resonant electromagnetic recognition at specific frequency. The next step is to test this hypothesis on an example of remdesivir drug as small molecule, which has been already approved for treatment of retroviruses. Remdesivir inhibits production of viral RNA and the mechanism how it is happening is not yet well explained. Some of its metabolites are interfering with viral RNAdependent RNA polymerase, while remdesivir can interact with viral protease [32], which interaction we have analyzed here. Activity of retroviral proteases is one of the critical steps in viral replication as this enzyme cleaves newly synthetized viral poly proteins to create mature protein components of an infectious virion. We have previously analyzed retroviral protease proteins and we have identified common RRM characteristic frequency at $\mathrm{fr}=0.0586 \pm 0.0040$ as described in reference [33] and presented in Figure 3.

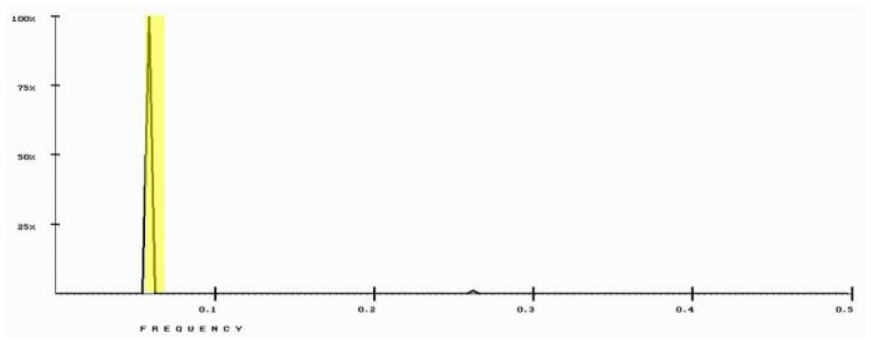

Figure 3. RRM cross-spectrum of retroviral protease proteins. The common RRM characteristic frequency is at $\mathrm{fr}=0.0586 \pm 0.0040$. RRM frequency range for remdesivir small molecule between 0.057-0.065 highlighted in yellow.

We have tested if remdesivir, as already approved anti-retroviral drug, can interact with retroviral protease through energy resonances as proposed above. Using the chemical formula for remdesivir $\left(\mathrm{C}_{27} \mathrm{H}_{35} \mathrm{~N}_{6} \mathrm{O}_{8} \mathrm{P}\right)$ as small molecules, we have calculated energy of free electrons within these small molecules to be at $\mathrm{Er}=0.0191 \mathrm{Ry}$. For this energy and refraction index for proteins, between 1.36-1.55 [25], we have calculated the corresponding RRM frequencies to be for remdesivir small molecule between 0.057-0.065, as highlighted in yellow in Figure 3. All intermediate calculations for remdesivir small molecule using formulas presented within Methods section have been presented within Table 1. The RRM frequency range for remdesivir overlaps characteristic RRM frequency for retroviral protease at $\mathrm{fr}=0.0586 \pm 0.0040$. As there is common frequency between characteristic RRM frequency of retroviral proteases and RRM frequency for remdesivir small molecules, we propose that remdesivir, as an approved antiretroviral drug is acting on retroviral proteases through energy resonance at RRM frequency $\mathrm{fr}=0.0586 \pm 0.0040$. This result is an example of how our hypothesis, that interaction between small molecules and related receptors/proteins is based on resonant electromagnetic recognition at specific frequency, can be used in future for preselection of medical drugs and better understanding of their activity as well.

\section{Interaction between Hydroxychloroquine and SARS-CoV-2 Spike Proteins}

SARS-CoV-2 spike proteins, which are on the surface of coronavirus, are the first to approach and recognize host cells [34]. Thus, we have analyzed spike proteins from different coronaviruses with the aim to find out if there is any common component that can characterize spikes recognition and interaction with host cells. When we have analyzed spike proteins from different coronaviruses, from UniProt database, as listed in Appendix, using the RRM, the most prominent common RRM frequency has been found at $f h=0.2827+0.0009$, as presented in Figure 4.

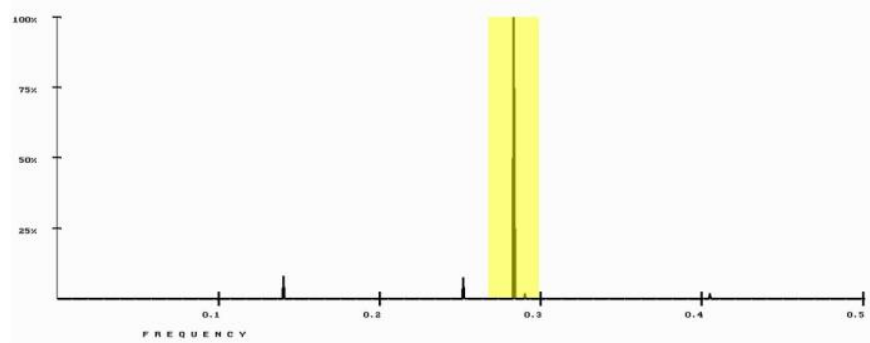

Figure 4. RRM cross-spectrum of spike proteins. The common RRM characteristic frequency is at $\mathrm{fh}=0.2827 \pm 0.0009$. RRM frequency range for hydroxychloroquine small molecule between 0.26 0.30 highlighted in yellow.

It is interesting to note that there is one unique common characteristic for all analyzed spike proteins from many different coronaviruses. This 
would mean that all coronaviruses have one and the same RRM characteristic frequency fh $=0.2827 \pm 0.0009, \quad$ characterizing viral recognition and interaction with host cells. Thus, there is possibility to interfere with frequency fh and prevent the very first step of viral infection by using small molecule drugs. One of such candidates is hydroxychloroquine, which has been already clinically tested for treatment of COVID19 patients [1-2].

Using the chemical formula for hydroxychloroquine small molecule $\left(\mathrm{C}_{18} \mathrm{H}_{26} \mathrm{~N}_{3} \mathrm{ClO}\right)$, we have calculated, as described in Methods section, energy of free electrons within hydroxychloroquine small molecule to be at Eh=0.0874Ry. For this energy and refraction index for proteins, which is between 1.36-1.55 [25], we have calculated the corresponding RRM frequency for hydroxychloroquine small molecule to be between $0.26-0.30$, as highlighted in yellow in Figure 4. All intermediate calculations for hydroxychloroquine small molecule using formulas presented within Methods section have been presented within Table 1 below. Strikingly, this RRM frequency range for hydroxychloroquine small molecule overlaps characteristic RRM frequency for SARS-CoV-2 spike proteins, which is at $\mathrm{fh}=0.2827 \pm 0.0009$. As there is overlap between characteristic RRM frequency fh for coronavirus spike proteins including SARS-CoV-2 and RRM frequency for hydroxychloroquine small molecules, we propose that hydroxychloroquine could interact with SARS-CoV-2 spike proteins and thus could be efficient in the first phase of infection by neutralising activity of spike proteins on the surface of coronavirus and thus preventing initial viral infection.

\section{Interaction between Chloroquine and COVID- 19 Viral Entry into Host Cells}

The next step of viral infection is interaction between coronavirus spike S1 fragments and receptors on the surface of host cells. The main entry point into host cells for some coronaviruses, including $\mathrm{HCoV}-\mathrm{NL63}$, SARS-CoV (the virus that causes SARS) [35] and SARS-CoV-2 (the virus that causes COVID-19) is angiotensin-converting enzyme 2 (ACE2), which is attached to outer surface of cell membranes of cells in the lungs, arteries, heart, kidney and intestines [36]. More specifically, the binding of the spike $\mathrm{S} 1$ protein fragments of SARS-CoV and SARS-CoV-2 to the enzymatic domain of ACE2 on the surface of cells results in endocytosis and translocation of both the virus and the enzyme into endosomes located within cells [34].
When we have analyzed spike S1 fragments and ACE2 receptors, from UniProt database, as listed in Appendix, the prominent common characteristic frequency appears to be at $f c=0.3145 \pm 0.0019$, characterising their mutual interaction, as presented in Figure 5 [12].

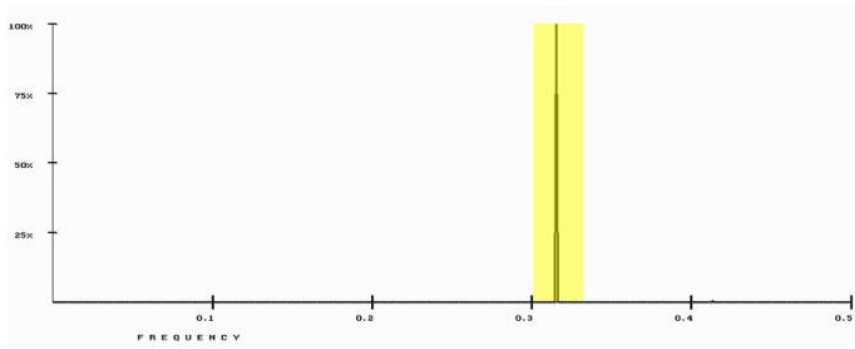

Figure 5. RRM cross-spectrum of spike S1 fragments from coronaviruses affecting humans and ACE2 receptors with common characteristic frequency of $\mathrm{fc}=0.3145 \pm 0.0019$. RRM frequency range for chloroquine small molecule between $0.30-0.33$ highlighted in yellow.

In addition, we have proposed that there is additional substrate for S1 spikes RBC Band3, which is on the surface of red blood cells [12]. When S1 spikes have been compared with RBC Band 3 proteins the same common characteristic frequency $\mathrm{fc}=0.3145 \pm 0.0019$ appears characterizing their interaction. We have speculated that this viral interaction with RBC Band 3 proteins on red blood cells is the cause of hypoxia in severe cases of COVID-19 [12]. It is interesting to note that $\mathrm{RBC}$ Band3 protein has been already found to be entry point for malaria parasite agents, merozoites from plasmodium falciparum into red blood cells [37] and this could be the reason why malaria drugs could be efficient in treatment of COVID-19. Thus, we have analysed chloroquine, approved malaria drug, as possible small molecule that can interfere with viral interaction with the cell either through ACE2 or RBC Band3.

Using the chemical formula for chloroquine small molecule $\left(\mathrm{C}_{18} \mathrm{H}_{26} \mathrm{~N}_{3} \mathrm{Cl}\right)$, we have calculated energy of free electrons within chloroquine small molecule to be at Ec=0.0946Ry. The interaction between spike $\mathrm{S} 1$ fragments and corresponding cell membrane receptors occurs within cell membrane, so for further calculations we have used the refraction index for cell membranes, which is between 1.46-1.60 [25]. For energy Ec and refraction index for cell membrane, we have calculated the corresponding RRM frequency for chloroquine small molecule to be between 0.30 0.33 , as highlighted in yellow in Figure 5. All 
intermediate calculations for chloroquine small molecule using formulas presented within Methods section have been presented within Table 1 below. Strikingly again, this RRM frequency range for chloroquine small molecule overlaps characteristic RRM frequency at $\mathrm{fc}=0.3145 \pm 0.0019, \quad$ for $\mathrm{S} 1$ fragments of coronavirus spike proteins (including SARS-CoV2) and their interaction with ACE2 receptors and/or RBC Band3 proteins. As this frequency fc, which is characterizing interaction between SARSCoV-2 S1 fragments with ACE2 receptors and/or RBC Band3 proteins, overlaps with RRM frequency for chloroquine small molecules, we propose that chloroquine could interfere with this interaction and thus could prevent viral entry into the host cells.

\section{Results Summary}

The results for all examples above have been summarized within Table 1, with all intermediate calculations for small molecules using formulas presented in Methods section, as well as related protein's RRM characteristic frequencies.

\begin{tabular}{|l|l|l|l|l|c|l|}
\hline $\begin{array}{l}\text { small } \\
\text { molecule }\end{array}$ & $\begin{array}{l}\text { average } \\
\text { valence } Z\end{array}$ & $\begin{array}{l}\text { free electrons } \\
\text { energy } E[R y]\end{array}$ & $\begin{array}{l}\text { refraction } \\
\text { index range }\end{array}$ & $\begin{array}{l}\text { wavelength } \\
\text { range } \lambda_{b}[\mathrm{~nm}]\end{array}$ & $\begin{array}{l}\text { frequency } \\
\text { range } \mathrm{f}_{5 m}\end{array}$ & $\begin{array}{l}\text { related protein } \\
\text { RRM frequency }\end{array}$ \\
\hline $\begin{array}{l}\text { cannabinoid } \\
\left(\mathrm{C}_{21} \mathrm{H}_{30} \mathrm{O}_{2}\right)\end{array}$ & 2.377 & 0.0942 & $1.46-1.60$ & $604-665$ & $0.30-0.33$ & $0.3174 \pm 0.0024$ \\
\hline $\begin{array}{l}\text { kainic acid } \\
\left(\mathrm{C}_{10} \mathrm{H}_{15} \mathrm{NO}_{4}\right)\end{array}$ & 2.800 & 0.0304 & $1.46-1.60$ & $1863-2062$ & $0.10-0.11$ & $0.1143 \pm 0.0008$ \\
\hline $\begin{array}{l}\text { remdesivir } \\
\left(\mathrm{C}_{27} \mathrm{H}_{35} \mathrm{~N}_{6} \mathrm{O}_{8} \mathrm{P}\right)\end{array}$ & 2.935 & 0.0191 & $1.36-1.55$ & $3072-3524$ & $0.057-0.065$ & $0.0586 \pm 0.0040$ \\
\hline $\begin{array}{l}\text { hydroxychloroquine } \\
\left(\mathrm{C}_{18} \mathrm{H}_{26} \mathrm{~N}_{3} \mathrm{ClO}\right)\end{array}$ & 2.571 & 0.0874 & $1.36-1.55$ & $651-717$ & $0.26-0.30$ & $0.2827 \pm 0.0009$ \\
\hline $\begin{array}{l}\text { chloroquine } \\
\left(\mathrm{C}_{18} \mathrm{H}_{26} \mathrm{~N}_{3} \mathrm{Cl}\right)\end{array}$ & 2.500 & 0.0946 & $1.46-1.60$ & $621-710$ & $0.30-0.33$ & $0.3145 \pm 0.0019$ \\
\hline
\end{tabular}

Table 1. Summarized results for all examples with all intermediate calculations using formulas presented in Methods section and characteristic RRM frequency for related proteins.

\section{Discussion and Conclusion}

With the rapid development of biotechnology and specially pharmacology, there is busy time for drug and medication developments. Lot of those developments are based on trial and error approach within biochemical experiments, which are time and resource consuming, as well as costly. So, there is large demand for computational rational drug preselection and design models. The most of existing computational models are based on 3D and electrostatic fit (docking) between small molecule and related substrate. Such approaches are not considering general functionality of substrates with similar biological function, nor long distance specific recognition between small molecules and their substrates. Here, we propose the completely new approach for preselection of bioactive drugs (small molecules) based on electromagnetic energy resonances between small molecules and substrates/proteins considering long distance selective resonance, as well as common biological functionality of related substrates/proteins.

We have previously established Resonant Recognition Model (RRM), which proposes that selectivity of biological interactions and functions between proteins, DNA/RNA, is based on resonant electromagnetic energy between interacting macromolecules at the specific frequency for the specific interaction/function. This RRM frequency is related to periodicities (frequencies) of free electron energies along protein, DNA/RNA macromolecules. However, this approach cannot be applied for interactions between proteins and small molecules, as small molecules are not linear sequential molecules, although they still selectively interact with proteins. Thus, we propose that small molecules selective interactions with proteins are also based on electromagnetic resonant recognition. Here, we have expanded the RRM model with the idea of selective electromagnetic resonant recognition to interaction of small molecules with proteins. For that purpose, we propose that energies of free electrons in small molecules are the most relevant for such resonant energy recognition. Thus, we have here calculated energies of free electrons within the small molecule and corresponding wavelength using quantum mechanics de Broglie formula. These wavelengths (frequencies) have been compared with RRM characteristic frequencies for relevant interacting proteins with the idea of checking if calculated frequencies for small molecules and interacting proteins are overlapping and enabling resonant recognition.

To support this idea, we have presented here two natural examples of small molecules interactions with related receptors, namely: cannabinoids with cannabinoid receptors, kainic acid (kainate) with glutamate kainic receptors as well as application to already approved drugs, which are also potential COVID-19 drugs including remdesivir, chloroquine and hydroxychloroquine for their ability to interact with viral proteins and thus interfere with viral infection. All these examples have shown that electromagnetic frequencies of free electrons within 
the small molecules are overlapping the characteristic RRM frequencies of related receptors/proteins, indicating that interactions between small molecules and related receptors/proteins are indeed based on resonant electromagnetic energy at specific frequency for specific interaction.

In case of remdesivir, which is already approved as retroviral drug, we found that it can interact, based on extended RRM using resonant energy transfer, with viral protease. Remdesivir inhibits production of viral RNA and the mechanism how it is happening is not yet well explained. Some of its metabolites are interfering with viral RNA-dependent RNA polymerase, but there are also predictions that remdesivir can interact with viral protease [32], which we have analyzed here. Activity of retroviral proteases is one of the critical steps in viral replication as this enzyme cleaves newly synthetized viral poly proteins to create mature protein components of an infectious virion.

In case of hydroxychloroquine, we have found that RRM frequency corresponding to energy of free electrons within hydroxychloroquine corresponds to RRM frequency characterizing coronavirus spike proteins including SARS-CoV-2. Thus, we propose that hydroxychloroquine could interfere with activity of coronavirus spike proteins and as such could be efficient treatment for the first phase of SARS-CoV-2 infection by neutralising activity of spike proteins on the surface of coronavirus and preventing initial viral infection.

In case of chloroquine, we have found that RRM frequency corresponding to energy of free electrons within chloroquine corresponds to RRM frequency characterizing interaction between $\mathrm{S} 1$ fragments of coronavirus spike proteins including SARS-CoV-2 and related receptors. Thus, we propose that chloroquine could impede interaction between S1 fragments of coronavirus spike proteins and related receptors, preventing viral entry into the host cells and as such could be considered as efficient treatment for COVID-19 in later stages of infection.

Interestingly, in all three cases of proposed drugs for treatment of COVID-19 (hydroxychloroquine, chloroquine, remdesivir), we have found absolute overlap between RRM characteristic frequencies of corona viral proteins and characteristic frequencies of related drugs, indicating that in all three cases the proposed drugs could be efficient for COVID-19 treatments, but in different ways through interactions with different viral proteins at different stages of COVID-19 viral infection.
The presented extension of the RRM model is aimed generally to aid developments within pharmaceutical industry towards finding the whole variety of effective drugs (small molecules), satisfying demand for pre-selective computational methods capable of quick preselection of small molecules before they are chemically and biologically tested. We have here proposed the extension of RRM model, which represents quick preselection method that could significantly narrow down number of experimentally tested potential molecular candidates and thus would enormously save resources, time and costs involved in discovery of new drugs, and consequently would provide quicker and better health outcomes.

Bearing in mind all the above, the extended RRM model is opening completely new avenues towards, not only understanding protein, DNA/RNA selectivity of interactions, but also understanding selectivity of interactions between small molecules and related receptors/proteins. With such understanding, we are opening new horizons for pharmaceutical industry, drug design and biochemistry in general.

\section{Contributions}

Conceptualization, I.C.; Methodology, I.C. and D.C.; Software, D.C.; Resources I.L.; Writing-Original Draft PreparationReview and Editing, I.C., D.C. and I.L.

\section{Competing Interests}

Authors declare they have no competing interests.

\section{Funding}

This research received no external funding.

\section{Acknowledgement}

The authors would like to thank Mr. Anthony Slingsby for proofreading this manuscript.

\section{References}

1. Pastick KA et al.: Review: Hydroxychloroquine and Chloroquine for Treatment of SARS-CoV-2 (COVID-19). Open Forum Infectious Diseases, 2020; 1-9.

2. Arshad S et al.: Treatment with Hydroxychloroquine, Azithromycin and Combination in Patients Hospitalized with COVID-19. International Journal of Infectious Diseases, 2020; 97, 396-403, doi: 10.1016/j.ijid.2020.06.099.

3. Tonnesmann E, Kandolf R, Lewalter T: Chloroquine Cardiomyopathy - a Review of the Literature. Immunopharmacol Immunotoxicol, 2013; 35:434-442.

4. Ben-Zvi I, Kivity S, Langevitz P, Shoenfeld Y: Hydroxychloroquine: from Malaria to Autoimmunity. Cliv Rev Allergy Immunol, 2012; 42:145-153.

5. Cosic I: Macromolecular Bioactivity: Is it Resonant Interaction between Macromolecules? -Theory and Applications. IEEE Trans on Biomedical Engineering, 1994; 41, 1101-1114.

6. Cosic I: The Resonant Recognition Model of Macromolecular Bioactivity: Theory and Applications. Basel: Birkhauser Verlag, 1997.

7. Cosic I: Resonant Recognition Model of Protein-Protein and Protein-DNA Recognition, in Bioinstrumentation and Biosensors. Marcel Dekker Inc New York, 1990; 475-510. 
8. Cosic I, Paspaliaris V, Cosic D: Biophysical Insights into Cystic Fibrosis Based on Electromagnetic Resonances in CFTR Proteins, International Journal of Sciences, 2019; 8(9), 1-8, doi: 10.18483/ijSci.2148.

9. Cosic I, Lazar K, Cosic D: Cellular Ageing - Telomere, Telomerase and Progerin analysed using Resonant Recognation Model. MD-Medical Data, 2014; 6(3), 205-209.

10. Cosic I, Cosic D, Lazar K: Analysis of Tumor Necrosis Factor Function Using the Resonant Recognition Model. Cell Biochemistry and Biophysics, 2015; doi: 10.1007/s12013015-0716-3.

11. Cosic I, Paspaliaris V, Cosic D: Analysis of Protein-Receptor on an Example of Leptin-Leptin Receptor Interaction Using the Resonant Recognition Model. Appl. Sci., 2019; 9, 5169, doi: 10.3390/app9235169.

12. Cosic I, Cosic D, Loncarevic I: RRM Prediction of Erythrocyte Band3 Protein as Alternative Receptor for SARS-CoV-2. MDPI Appl. Sci., 2020; 10, 4053, doi: 10.3390/app10114053.

13. Cosic I, Lazar K, Cosic D: Prediction of Tubulin Resonant Frequencies Using the Resonant Recognition Model (RRM). IEEE Trans. on NanoBioscience, 2015; 12, 491-496; doi: 10.1109/TNB.2014.2365851.

14. Cosic I, Cosic D, Lazar K: Is It Possible to Predict Electromagnetic Resonances in Proteins, DNA and RNA? Nonlinear Biomedical Physics, 2015; 3, doi: 10.1140/s40366015-0020-6.

15. Cosic I, Cosic D: The Treatment of Crigler-Najjar Syndrome by Blue Light as Explained by Resonant Recognition Model. EPJ Nonlinear Biomedical Physics, 2016; 4(9), doi: 10.1140/epjnbp/s40366-016-0036-6.

16. Cosic I, Cosic D, Lazar K: Environmental Light and Its Relationship with Electromagnetic Resonances of Biomolecular Interactions, as Predicted by the Resonant Recognition Model. International Journal of Environmental Research and Public Health, 2016; 13(7), 647, doi: 10.3390/ijeprh13070647.

17. Cosic I, Paspaliaris V, Cosic D: Explanation of Osteoblastic Differentiation of Stem Cells by Photo Biomodulation Using the Resonant Recognition Model. Appl. Sci., 2019; 9, 1979, doi: 10.3390/app9101979.

18. Vojisavljevic V, Pirogova E, Cosic I: The Effect of Electromagnetic Radiation $(550 \mathrm{~nm}-850 \mathrm{~nm})$ on I-Lactate Dehydrogenase Kinetics. Internat J Radiat Biol, 2007; 83, 221-230.

19. Dotta BT, Murugan NJ, Karbowski LM, Lafrenie RM, Persinger MA: Shifting Wavelength of Ultraweak Photon Emissions from Dying Melanoma Cells: Their Chemical Enhancement and Blocking Are Predicted by Cosic's Theory of Resonant Recognition Model for Macromolecules. Naturwissenschaften, 2014; 101(2), doi: 10.1007/s00114013-1133-3.

20. Murugan NJ, Karbowski LM, Persinger MA: Cosic's Resonance Recognition Model for Protein Sequences and Photon Emission Differentiates Lethal and Non-Lethal Ebola Strains: Implications for Treatment. Open Journal of Biophysics, 2014; 5, 35.

21. Karbowski LM, Murugan NJ, Persinger MA: Novel Cosic Resonance (Standing Wave) Solutions for Components of the JAK-STAT Cellular Signalling Pathway: A Convergence of Spectral Density Profiles. FEBS Open Bio, 2015; 5, 245-250.

22. Veljkovic V, Slavic I: General Model od Pseudopotentials. Physical Review Letters, 1972; 29, 105-108.

23. Veljkovic V: A Theoretical Approach to Preselection of Cancerogens and Chemical Carcinogenesis. Gordon \& Breach New York, 1980.

24. Veljkovic V: The Dependence of the Fermi Energy on the Atomic Number, Physics Letters, 1973; 45A(1), 41-42.

25. Mohsin ASM, Salim MB: Probing the Intracellular Refractive Index and Molecular Interaction of Gold Nanoparticles in HeLa Cells Using Single Particle
Spectroscopy. International Journal of Nanomedicine, 2018; 13, 6019-6028, doi: 10.2147/IJN.S175523.

26. Aizpurua-Olaizola O, Elezgarai I, Rico-Bario I, Zarandona I, Etxebarria N, Usobiaga A: Targeting the Endocannabinoid System: Future Therapeutic Strategies. Drug Discovery Today, 2016; doi: 10.1016/j.drudis.2016.08.005.

27. Howlett AC: The Cannabinoid Receptors. Prostaglandins Other Lipid Mediat., 2002; 68-69, 619-631, doi: 10.1016/S0090-6980(02)00060.

28. Brassai A, Suvanjeiev RG, Bán EG, Lakatos M: Role of Synaptic and Nonsynaptic Glutamate Receptors in Ischaemia Induced Neurotoxicity. Review. Brain Research Bulletin, 2015; 112, 1-6, doi: 10.1016/j.brainresbull.2014.12.007.

29. Steinhäuse C, Gallo V: News on Glutamate Receptors in Glial Cells. Trends Neurosci, 1996; 19(8), 339-345, doi: 10.1016/0166-2236(96)10043-6.

30. Teichberg VI: Glial Glutamate Receptors: Likely Actors in Brain Signalling. FASEB J, 1991; 5(15), 3086-3091, doi: 10.1096/fasebj.5.15.1660422.

31. Huettner JE: Kainate Receptors and Synaptic Transmission. Progress in Neurobiology, 2003; 70(5), 387-407, doi 10.1016/S0301-0082(03)00122-9.

32. Naik VR, Munikumar M, Ramakrishna U, Srujana M, Goudar G, Naresh P, Kumar BN, Hemalatha R: Remdesivi (GS-5734) as a Therapeutic Option of 2019-nCOV Main Protease - In Silico Approach. J Biomol Struct Dyn, 2020; 114, doi: 10.1080/07391102.2020.1781694.

33. Caceres JLH, Cosic I, Cosic D: Retroviral Proteases Viewed through the Resonant Recognition Model. MD-Medical Data, 2014; 6(2), 117-123.

34. Li F: Receptor Recognition and Cross-species Infections of SARS Coronavirus. Antiviral Research, 2013; 100(1), 24654, doi: 10.1016/j.antiviral.2013.08.014.

35. Xu X, Chen P, Wang J, Feng J, Zhou H, Li X et al.: Evolution of the Novel Coronavirus from the Ongoing Wuhan Outbreak and Modelling of its Spike Protein for Risk of Human Transmission. Science China. Life Sciences, 2020; 63 (3), 457-460, doi: 10.1007/s11427-020-1637-5.

36. Millet JK: Whittaker G.R. Physiological and Molecular Triggers for SARS-CoV Membrane Fusion and Entry into Host Cells. Virology, 2018; 517, 3-8. doi 10.1016/j.virol.2017.12.015.

37. Perkins ME: Binding of Glycophorins to Plasmodium Falciparum Merozoites. Molecular and Biochemical Parasitology, 1984; 10(1), 67-78

\section{Appendix}

The protein sequences from UniProt Database analyzed using the RRM model:

Cannabis receptors: P20272, P21554, P47746, P56971, O02777, Q5IS73, Q71SP5, P34972, Q9QZN9, P47936, Q9Y2T6, Q3UJF0, Q9Y2T5, O35433, Q704Y3, Q8NER1, Q6R5A3, Q6RX08, Q697L1, Q96F85, Q5M8N0, Q5M7A7, Q17QM9, Q9GKT6.

Glutamate kainic receptors: P39086, Q38PU4, Q60934, P22756, R8QF68, Q13002, Q38PU3, P39087, P42260, Q91755, Q13003, Q38PU2, B1AS29, P42264, Q16099, Q8BMF5, Q5IS46, Q01812, Q16478, Q61626, Q63273.

Cleaved, active spike proteins: K9N5Q8, Q5MQD0, Q14EB0, Q0ZME7, P36334, P59594,

P11224, P11225, Q8BB25, Q9IKD1, P05135, P11223, Q0Q466, P36300, Q65984, Q7T6T3, P15423, Q6Q1S2, P18450, P33470, P07946, P27655, P24413, Q01977, P10033, Q91AV1 and QHD43416.1.

S1 fragment spike proteins: P0DTC2, P15423, P36334, Q6Q1S2, P59594, K9N5Q8, Q0ZME7, Q5MQD0, Q14EB0.

ACE2 receptors: Q5EGZ1, Q56NL1, Q9BYF1, Q8R0I0, Q59RR0, P21192, Q56H28, Q58DD0, Q5RFN1, Q6FJQ9 